Pacific

Journal of

Mathematics

\title{
A NEW CHARACTERIZATION OF COMPLETE LINEAR WEINGARTEN HYPERSURFACES IN REAL SPACE FORMS
}

Cícero P. Aquino, Henrique F. De Lima and Marco A. L. Velásquez 


\title{
A NEW CHARACTERIZATION OF COMPLETE LINEAR WEINGARTEN HYPERSURFACES IN REAL SPACE FORMS
}

\author{
Cícero P. Aquino, Henrique F. de lima and Marco A. L. Velásquez
}

\begin{abstract}
We apply the Hopf's strong maximum principle in order to obtain a suitable characterization of the complete linear Weingarten hypersurfaces immersed in a real space form $\mathbb{Q}_{c}^{n+1}$ of constant sectional curvature $c$. Under the assumption that the mean curvature attains its maximum and supposing an appropriated restriction on the norm of the traceless part of the second fundamental form, we prove that such a hypersurface must be either totally umbilical or isometric to a Clifford torus, if $c=1$, a circular cylinder, if $c=0$, or a hyperbolic cylinder, if $c=-1$.
\end{abstract}

\section{Introduction and statement of the main result}

Many authors have approached the problem of characterizing hypersurfaces immersed with constant mean curvature or with constant scalar curvature in a real space form $\mathbb{Q}_{c}^{n+1}$ of constant sectional curvature $c$. In this setting, Cheng and Yau [1977] introduced a new self-adjoint differential operator $\square$ acting on smooth functions defined on Riemannian manifolds. As a byproduct of this approach they were able to classify closed hypersurfaces $M^{n}$ with constant normalized scalar curvature $R$ satisfying $R \geq c$ and nonnegative sectional curvature immersed in $\mathbb{Q}_{c}^{n+1}$. Later on, $\mathrm{Li}$ [1996] extended the results of Cheng and Yau in terms of the squared norm of the second fundamental form of the hypersurface $M^{n}$. Shu [2007] applied the generalized Omori-Yau maximum principle [Omori 1967; Yau 1975] to prove that a complete hypersurface $M^{n}$ in the hyperbolic space $\mathbb{\boxplus}^{n+1}$ with constant normalized scalar curvature and nonnegative sectional curvature must be either totally umbilical or isometric to a hyperbolic cylinder $\mathbb{W}^{1}\left(-\sqrt{1+r^{2}}\right) \times \mathbb{S}^{n-1}(r)$.

Li [1997] studied the rigidity of compact hypersurfaces with nonnegative sectional curvature immersed in a unit sphere with scalar curvature proportional to mean curvature. Next, Li et al. [2009] extended the result of [Cheng and Yau 1977; Li 1997] by considering linear Weingarten hypersurfaces immersed in the

MSC2010: primary 53C42; secondary 53A10, 53C20, 53C50.

Keywords: space forms, linear Weingarten hypersurfaces, totally umbilical hypersurfaces, Clifford torus, circular cylinder, hyperbolic cylinder. 
unit sphere $\mathbb{S}^{n+1}$, that is, hypersurfaces of $\mathbb{S}^{n+1}$ whose mean curvature $H$ and normalized scalar curvature $R$ satisfy $R=a H+b$, for some $a, b \in \mathbb{R}$. In this setting, they showed that if $M^{n}$ is a compact linear Weingarten hypersurface with nonnegative sectional curvature immersed in $\mathbb{S}^{n+1}$, such that $R=a H+b$ with $(n-1) a^{2}+4 n(b-1) \geq 0$, then $M^{n}$ is either totally umbilical or isometric to a Clifford torus $\mathbb{S}^{k}\left(\sqrt{1-r^{2}}\right) \times \mathbb{S}^{n-k}(r)$, where $1 \leq k \leq n-1$. Thereafter, Shu [2010] obtained some rigidity theorems concerning to linear Weingarten hypersurfaces with two distinct principal curvatures immersed in $\mathbb{Q}_{c}^{n+1}$.

In [Brasil et al. 2010], Brasil Jr., Colares and Palmas used the generalized maximum principle of Omori-Yau to characterize complete hypersurfaces with constant scalar curvature in $\mathbb{S}^{n+1}$. By applying a weak Omori-Yau maximum principle due to Pigola, Rigoli and Setti [Pigola et al. 2005], Alías and GarcíaMartínez [2010] studied the behavior of the scalar curvature $R$ of a complete hypersurface immersed with constant mean curvature into a real space form $\mathbb{Q}_{c}^{n+1}$, deriving a sharp estimate for the infimum of $R$. More recently, Alías, GarcíaMartínez and Rigoli [Alías et al. 2012] obtained another suitable weak maximum principle for complete hypersurfaces with constant scalar curvature in $\mathbb{Q}_{c}^{n+1}$, and gave some applications of it in order to estimate the norm of the traceless part of its second fundamental form. In particular, they extended the main theorem of [Brasil et al. 2010] for the context of $\mathbb{Q}_{c}^{n+1}$.

Here, our purpose is to establish a new characterization theorem concerning the complete linear Weingarten hypersurfaces immersed in a real space form $\mathbb{Q}_{c}^{n+1}$. Under the assumption that the mean curvature $H$ attains its maximum along the hypersurface $M^{n}$ and supposing an appropriated restriction on the norm of the traceless part $\Phi$ of the second fundamental form of $M^{n}$, we get the following theorem.

Theorem 1.1. Let $M^{n}$ be a complete linear Weingarten hypersurface immersed in a real space form $\mathbb{Q}_{c}^{n+1}, n \geq 3$, such that $R=a H+b$ with $b>c$. Suppose that $R>0$, when $c=0$ or $c=-1$, and that $R>(n-2) / n$, when $c=1$. If $H$ attains its maximum on $M^{n}$ and

$$
\sup _{M}|\Phi|^{2} \leq \frac{n(n-1) R^{2}}{(n-2)(n R-(n-2) c)},
$$

then either

i. $|\Phi| \equiv 0$ and $M^{n}$ is totally umbilical, or

ii. $|\Phi|^{2} \equiv \frac{n(n-1) R^{2}}{(n-2)(n R-(n-2) c)}$ and $M^{n}$ is isometric to

(a) a Clifford torus $\mathbb{S}^{1}\left(\sqrt{1-r^{2}}\right) \times \mathbb{S}^{n-1}(r)$, when $c=1$,

(b) a circular cylinder $\mathbb{R} \times \mathbb{S}^{n-1}(r)$, when $c=0$, or

(c) a hyperbolic cylinder $\mathbb{M}^{1}\left(-\sqrt{1+r^{2}}\right) \times \mathbb{S}^{n-1}(r)$, when $c=-1$, 
where in each case $r=\sqrt{\frac{n-2}{n R}}$.

The proof of Theorem 1.1 is given in Section 3, jointly with a corollary related to the compact case.

\section{Preliminaries}

In this section we will introduce some basic facts and notation that will appear on the paper. In what follows, we will suppose that all hypersurfaces are orientable and connect.

Let $M^{n}$ be an $n$-dimensional hypersurface in a real space form $\mathbb{Q}_{c}^{n+1}$. We choose a local field of orthonormal frame $\left\{e_{A}\right\}$ in $\mathbb{Q}_{c}^{n+1}$, with dual coframe $\left\{\omega_{A}\right\}$, such that, at each point of $M^{n}, e_{1}, \ldots, e_{n}$ are tangent to $M^{n}$ and $e_{n+1}$ is normal to $M^{n}$. We will use the following convention for the indices:

$$
1 \leq A, B, C, \ldots \leq n+1, \quad 1 \leq i, j, k, \ldots \leq n .
$$

In this setting, denoting by $\left\{\omega_{A B}\right\}$ the connection forms of $\mathbb{Q}_{c}^{n+1}$, we have that the structure equations of $\mathbb{Q}_{c}^{n+1}$ are given by

$$
\begin{gathered}
d \omega_{A}=\sum_{i} \omega_{A i} \wedge \omega_{i}+\omega_{A n+1} \wedge \omega_{n+1}, \quad \omega_{A B}+\omega_{B A}=0, \\
d \omega_{A B}=\sum_{C} \omega_{A C} \wedge \omega_{C B}-\frac{1}{2} \sum_{C, D} K_{A B C D} \omega_{C} \wedge \omega_{D}, \\
K_{A B C D}=c\left(\delta_{A C} \delta_{B D}-\delta_{A D} \delta_{B C}\right) .
\end{gathered}
$$

Next, we restrict all the tensors to $M^{n}$. First of all, $\omega_{n+1}=0$ on $M^{n}$, so $\sum_{i} \omega_{n+1 i} \wedge \omega_{i}=d \omega_{n+1}=0$ and by Cartan's Lemma [1938] we can write

$$
\omega_{n+1 i}=\sum_{j} h_{i j} \omega_{j}, \quad h_{i j}=h_{j i}
$$

This gives the second fundamental form of $M^{n}, B=\sum_{i j} h_{i j} \omega_{i} \omega_{j} e_{n+1}$. The mean curvature $H$ of $M^{n}$ is defined by $H=\frac{1}{n} \sum_{i} h_{i i}$.

The structure equations of $M^{n}$ are

$$
\begin{gathered}
d \omega_{i}=\sum_{j} \omega_{i j} \wedge \omega_{j}, \quad \omega_{i j}+\omega_{j i}=0, \\
d \omega_{i j}=\sum_{k} \omega_{i k} \wedge \omega_{k j}-\frac{1}{2} \sum_{k, l} R_{i j k l} \omega_{k} \wedge \omega_{l} .
\end{gathered}
$$

Using the structure equations we obtain the Gauss equation 


$$
R_{i j k l}=c\left(\delta_{i k} \delta_{j l}-\delta_{i l} \delta_{j k}\right)+\left(h_{i k} h_{j l}-h_{i l} h_{j k}\right),
$$

where $R_{i j k l}$ are the components of the curvature tensor of $M^{n}$.

The Ricci curvature and the normalized scalar curvature of $M^{n}$ are given, respectively, by

$$
\begin{gathered}
R_{i j}=(n-1) c \delta_{i j}+n H h_{i j}-\sum_{k} h_{i k} h_{k j}, \\
R=\frac{1}{n(n-1)} \sum_{i} R_{i i} .
\end{gathered}
$$

From (2-8) and (2-9) we obtain

$$
|B|^{2}=n^{2} H^{2}-n(n-1)(R-c),
$$

where $|B|^{2}=\sum_{i, j} h_{i j}^{2}$ is the square of the length of the second fundamental form $B$ of $M^{n}$.

Set $\Phi_{i j}=h_{i j}-H \delta_{i j}$. We will also consider the following symmetric tensor

$$
\Phi=\sum_{i, j} \Phi_{i j} \omega_{i} \omega_{j}
$$

Let $|\Phi|^{2}=\sum_{i, j} \Phi_{i j}^{2}$ be the square of the length of $\Phi$. It is easy to check that $\Phi$ is traceless and, from (2-10), we get

$$
|\Phi|^{2}=|B|^{2}-n H^{2}=n(n-1) H^{2}-n(n-1)(R-c) .
$$

The components $h_{i j k}$ of the covariant derivative $\nabla B$ satisfy

$$
\sum_{k} h_{i j k} \omega_{k}=d h_{i j}+\sum_{k} h_{i k} \omega_{k j}+\sum_{k} h_{j k} \omega_{k i} .
$$

The Codazzi equation and the Ricci identity are, respectively, given by

$$
\begin{gathered}
h_{i j k}=h_{i k j}, \\
h_{i j k l}-h_{i j l k}=\sum_{m} h_{m j} R_{m i k l}+\sum_{m} h_{i m} R_{m j k l},
\end{gathered}
$$

where $h_{i j k}$ and $h_{i j k l}$ denote the first and the second covariant derivatives of $h_{i j}$.

The Laplacian $\Delta h_{i j}$ of $h_{i j}$ is defined by $\Delta h_{i j}=\sum_{k} h_{i j k k}$. From (2-13) and (2-14), we obtain

$$
\Delta h_{i j}=\sum_{k} h_{k k i j}+\sum_{k, l} h_{k l} R_{l i j k}+\sum_{k, l} h_{l i} R_{l k j k} .
$$

Since $\Delta|B|^{2}=2\left(\sum_{i, j} h_{i j} \Delta h_{i j}+\sum_{i, j, k} h_{i j k}^{2}\right)$, from (2-15) we get

$$
\frac{1}{2} \Delta|B|^{2}=|\nabla B|^{2}+\sum_{i, i, k} h_{i j} h_{k k i j}+\sum_{i, j, k, l} h_{i j} h_{l k} R_{l i j k}+\sum_{i, j, k, l} h_{i j} h_{i l} R_{l k j k} .
$$


Consequently, taking a (local) orthonormal frame $\left\{e_{1}, \ldots, e_{n}\right\}$ on $M^{n}$ such that $h_{i j}=\lambda_{i} \delta_{i j}$, from (2-16) we obtain the following Simons-type formula

$$
\frac{1}{2} \Delta|B|^{2}=|\nabla B|^{2}+\sum_{i} \lambda_{i}(n H)_{, i i}+\frac{1}{2} \sum_{i, j} R_{i j i j}\left(\lambda_{i}-\lambda_{j}\right)^{2} .
$$

Let $\phi=\sum_{i, j} \phi_{i j} \omega_{i} \omega_{j}$ be a symmetric tensor on $M^{n}$ defined by $\phi_{i j}=n H \delta_{i j}-h_{i j}$. Following [Cheng and Yau 1977], we introduce a operator $\square$ associated to $\phi$ acting on any smooth function $f$ by

$$
\square f=\sum_{i, j} \phi_{i j} f_{i j}=\sum_{i, j}\left(n H \delta_{i j}-h_{i j}\right) f_{i j} .
$$

Since $\phi_{i j}$ is divergence-free, it follows from the same reference that the operator $\square$ is self-adjoint relative to the $L^{2}$ inner product of $M^{n}$, that is,

$$
\int_{M} f \square g=\int_{M} g \square f
$$

for any smooth functions $f$ and $g$ on $M^{n}$.

Now, setting $f=n H$ in (2-18) and taking a local frame field $\left\{e_{1}, \ldots, e_{n}\right\}$ on $M^{n}$ such that $h_{i j}=\lambda_{i} \delta_{i j}$, from (2-10) we obtain the following:

$$
\begin{aligned}
\square(n H) & =n H \Delta(n H)-\sum_{i} \lambda_{i}(n H)_{, i i} \\
& =\frac{1}{2} \Delta(n H)^{2}-\sum_{i}(n H)_{, i}^{2}-\sum_{i} \lambda_{i}(n H)_{, i i} \\
& =\frac{n(n-1)}{2} \Delta R+\frac{1}{2} \Delta|B|^{2}-n^{2}|\nabla H|^{2}-\sum_{i} \lambda_{i}(n H)_{, i i} .
\end{aligned}
$$

Hence, taking into account (2-17), we get

$$
\square(n H)=\frac{n(n-1)}{2} \Delta R+|\nabla B|^{2}-n^{2}|\nabla H|^{2}+\frac{1}{2} \sum_{i, j} R_{i j i j}\left(\lambda_{i}-\lambda_{j}\right)^{2} .
$$

\section{Proof of Theorem 1.1 and a corollary}

In order to prove our result, to use some auxiliary lemmas are necessary. The first is a classic algebraic lemma due to M. Okumura [1974], and completed with the equality case proved by H. Alencar and M. do Carmo [1994].

Lemma 3.1. Let $\mu_{1}, \ldots, \mu_{n}$ be real numbers such that $\sum_{i} \mu_{i}=0$ and $\sum_{i} \mu_{i}^{2}=\beta^{2}$, where $\beta \geq 0$. Then

$$
-\frac{n-2}{\sqrt{n(n-1)}} \beta^{3} \leq \sum_{i} \mu_{i}^{3} \leq \frac{n-2}{\sqrt{n(n-1)}} \beta^{3},
$$

and equality holds if and only if at least $n-1$ of the numbers $\mu_{i}$ are equal. 
To obtain the second lemma, we will reason as in the proof of Lemma 2.1 of [Li et al. 2009].

Lemma 3.2. Let $M^{n}$ be a linear Weingarten hypersurface in a space form $\mathbb{Q}_{c}^{n+1}$, such that $R=a H+b$ for some $a, b \in \mathbb{R}$. Suppose that

$$
(n-1) a^{2}+4 n(b-c) \geq 0 .
$$

Then

$$
|\nabla B|^{2} \geq n^{2}|\nabla H|^{2} .
$$

Moreover, if the inequality (3-2) is strict and equality holds in (3-3) on $M^{n}$, then $H$ is constant on $M^{n}$.

Proof. Since we are supposing that $R=a H+b$, from (2-10) we get

$$
2 \sum_{i, j} h_{i j} h_{i j k}=\left(2 n^{2} H-n(n-1) a\right) H_{, k} .
$$

Thus,

$$
4 \sum_{k}\left(\sum_{i, j} h_{i j} h_{i j k}\right)^{2}=\left(2 n^{2} H-n(n-1) a\right)^{2}|\nabla H|^{2} .
$$

Consequently, using the Cauchy-Schwartz inequality, we obtain

$$
\begin{aligned}
4|B|^{2}|\nabla B|^{2} & =4\left(\sum_{i, j} h_{i j}^{2}\right)\left(\sum_{i, j, k} h_{i j k}^{2}\right) \\
& \geq 4 \sum_{k}\left(\sum_{i, j} h_{i j} h_{i j k}\right)^{2}=\left(2 n^{2} H-n(n-1) a\right)^{2}|\nabla H|^{2} .
\end{aligned}
$$

On the other hand, since $R=a H+b$, from (2-10) we easily see that

$$
\left(2 n^{2} H-n(n-1) a\right)^{2}=n^{2}(n-1)\left((n-1) a^{2}+4 n(b-c)\right)+4 n^{2}|B|^{2} .
$$

Hence, from (3-4) we have

$$
|B|^{2}|\nabla B|^{2} \geq n^{2}|B|^{2}|\nabla H|^{2} .
$$

Therefore, we obtain either $|B|=0$ and $|\nabla B|^{2}=n^{2}|\nabla H|^{2}$, or $|\nabla B|^{2} \geq n^{2}|\nabla H|^{2}$. Moreover, if $(n-1) a^{2}+4 n(b-c)>0$, from the previous identity we get that $\left(2 n^{2} H-n(n-1) a\right)^{2}>4 n^{2}|B|^{2}$. Now, let us assume in addition that the equality holds in (3-3) on $M^{n}$. In this case, we wish to show that $H$ is constant on $M^{n}$. Suppose, by way of contradiction, that it does not occur. Consequently, there exists a point $p \in M^{n}$ such that $|\nabla H(p)|>0$. So, one deduces from (3-4) that

$$
4|B(p)|^{2}|\nabla B(p)|^{2}>4 n^{2}|B(p)|^{2}|\nabla H(p)|^{2}
$$


and, since $|\nabla B(p)|^{2}=n^{2}|\nabla H(p)|^{2}>0$, we arrive at a contradiction. Hence, in this case, we conclude that $H$ must be constant on $M^{n}$.

In what follows, we will consider the Cheng-Yau modified operator

$$
L=\square-\frac{n-1}{2} a \Delta .
$$

Related to operator, we have the following sufficient criterion for ellipticity.

Lemma 3.3. Let $M^{n}$ be a linear Weingarten hypersurface immersed in a space form $\mathbb{Q}_{c}^{n+1}$, such that $R=a H+b$ with $b>c$. Then, $L$ is elliptic.

Proof. From (2-10), since $R=a H+b$ with $b>c$, we easily see that $H$ can not vanish on $M^{n}$ and, by choosing the appropriate Gauss mapping, we may assume that $H>0$ on $M^{n}$.

Let us consider the case that $a=0$. Since $R=b>c$, from (2-10) if we choose a (local) orthonormal frame $\left\{e_{1}, \ldots, e_{n}\right\}$ on $M^{n}$ such that $h_{i j}=\lambda_{i} \delta_{i j}$, we have $\sum_{i<j} \lambda_{i} \lambda_{j}>0$. Consequently,

$$
n^{2} H^{2}=\sum_{i} \lambda_{i}^{2}+2 \sum_{i<j} \lambda_{i} \lambda_{j}>\lambda_{i}^{2}
$$

for every $i=1, \ldots, n$ and, hence, we have that $n H-\lambda_{i}>0$ for every $i$. Therefore, in this case, we conclude that $L$ is elliptic.

Now, suppose $a \neq 0$. From (2-10) we get that

$$
a=-\frac{1}{n(n-1) H}\left(|B|^{2}-n^{2} H^{2}+n(n-1)(b-c)\right) .
$$

Hence, for every $i=1, \ldots, n$, a straightforward algebraic computation yields

$$
\begin{aligned}
n H-\lambda_{i}-\frac{n-1}{2} a & =n H-\lambda_{i}+\frac{1}{2 n H}\left(|B|^{2}-n^{2} H^{2}+n(n-1)(b-c)\right) \\
& =\frac{1}{2 n H}\left(\sum_{j \neq i} \lambda_{j}^{2}+\left(\sum_{j \neq i} \lambda_{j}\right)^{2}+n(n-1)(b-c)\right) .
\end{aligned}
$$

Therefore, since $b>c$, we also conclude in this case that $L$ is elliptic.

Proof of Theorem 1.1. Choose a (local) orthonormal frame $\left\{e_{1}, \ldots, e_{n}\right\}$ on $M^{n}$ such that $h_{i j}=\lambda_{i} \delta_{i j}$. Since $R=a H+b$, from (2-19) and (3-5) we have

$$
L(n H)=|\nabla B|^{2}-n^{2}|\nabla H|^{2}+\frac{1}{2} \sum_{i, j} R_{i j i j}\left(\lambda_{i}-\lambda_{j}\right)^{2} .
$$

Thus, since from (2-7) we have $R_{i j i j}=\lambda_{i} \lambda_{j}+c$, we get from (3-6)

$$
L(n H)=|\nabla B|^{2}-n^{2}|\nabla H|^{2}+n c\left(|B|^{2}-n H^{2}\right)-|B|^{4}+n H \sum_{i} \lambda_{i}^{3} .
$$


Moreover, we have $\Phi_{i j}=\mu_{i} \delta_{i j}$ and, with a straightforward computation, we verify that

$$
\sum_{i} \mu_{i}=0, \quad \sum_{i} \mu_{i}^{2}=|\Phi|^{2} \quad \text { and } \quad \sum_{i} \mu_{i}^{3}=\sum_{i} \lambda_{i}^{3}-3 H|\Phi|^{2}-n H^{3} .
$$

Thus, using Gauss (2-7) jointly with (3-8) into (3-7), we get

$$
L(n H)=|\nabla B|^{2}-n^{2}|\nabla H|^{2}+n H \sum_{i} \mu_{i}^{3}+|\Phi|^{2}\left(-|\Phi|^{2}+n H^{2}+n c\right) .
$$

By applying Lemmas 3.1 and 3.2, from (3-9) we have

$$
L(n H) \geq|\Phi|^{2}\left(-|\Phi|^{2}-\frac{n(n-2)}{\sqrt{n(n-1)}} H|\Phi|+n H^{2}+n c\right) .
$$

On the other hand, from (2-11), we obtain

$$
H^{2}=\frac{1}{n(n-1)}|\Phi|^{2}+(R-c)
$$

Thus, from (3-10) and (3-11) we get

$$
L(H) \geq \frac{1}{n(n-1)}|\Phi|^{2} P_{R}(|\Phi|),
$$

where

$$
P_{R}(x)=-(n-2) x^{2}-(n-2) x \sqrt{x^{2}+n(n-1)(R-c)}+n(n-1) R .
$$

Since we are supposing that $R>0, P_{R}(0)=n(n-1) R>0$ and the function $P_{R}(x)$ is strictly decreasing for $x \geq 0$, with $P_{R}\left(x^{*}\right)=0$ at

$$
x^{*}=R \sqrt{\frac{n(n-1)}{(n-2)(n R-(n-2) c)}}>0 .
$$

Thus, the hypothesis (1-1) guarantees that

$$
L(H) \geq \frac{1}{n(n-1)}|\Phi|^{2} P_{R}(|\Phi|) \geq 0 .
$$

Consequently, since Lemma 3.3 guarantees that $L$ is elliptic and as we are supposing that $H$ attains its maximum on $M^{n}$, from (3-13) we conclude that $H$ is constant on $M^{n}$. Thus, taking into account (3-6), we get

$$
|\nabla B|^{2}=n^{2}|\nabla H|^{2}=0,
$$

and it follows that $\lambda_{i}$ is constant for every $i=1, \ldots, n$.

If $|\Phi|<x^{*}$, then from (3-13) we have that $|\Phi|=0$ and, hence, $M^{n}$ is totally umbilical. If $|\Phi|=x^{*}$, since the equality holds in (3-1) of Lemma 3.1, we conclude that $M^{n}$ is either totally umbilical or an isoparametric hypersurface with two distinct principal curvatures one of which is simple. 
Hence, by the classical results on isoparametric hypersurfaces of real space forms [Cartan 1938; Levi-Civita 1937; Segre 1938] and since we are supposing $R>0$, we conclude that either $|\Phi|=0$ and $M^{n}$ is totally umbilical, or

$$
|\Phi|^{2}=\frac{n(n-1) R^{2}}{(n-2)(n R-(n-2) c)}
$$

and $M^{n}$ is isometric to

(a) a Clifford torus $\mathbb{S}^{1}\left(\sqrt{1-r^{2}}\right) \times \mathbb{S}^{n-1}(r)$, with $0<r<1$, if $c=1$,

(b) a circular cylinder $\mathbb{R} \times \mathbb{S}^{n-1}(r)$, with $r>0$, if $c=0$, or

(c) a hyperbolic cylinder $\mathbb{H}^{1}\left(-\sqrt{1+r^{2}}\right) \times \mathbb{S}^{n-1}(r)$, with $r>0$, if $c=-1$.

When $c=1$, for a given radius $0<r<1$, is a standard fact that the product embedding $\mathbb{S}^{1}\left(\sqrt{1-r^{2}}\right) \times \mathbb{S}^{n-1}(r) \hookrightarrow \mathbb{S}^{n+1}$ has constant principal curvatures given by

$$
\lambda_{1}=\frac{r}{\sqrt{1-r^{2}}}, \quad \lambda_{2}=\cdots=\lambda_{n}=-\frac{\sqrt{1-r^{2}}}{r} .
$$

Thus, in this case,

$$
H=\frac{n r^{2}-(n-1)}{n r \sqrt{1-r^{2}}} \quad \text { and } \quad|\Phi|^{2}=\frac{n-1}{n r^{2}\left(1-r^{2}\right)} .
$$

When $c=0$, for a given radius $r>0, \mathbb{R} \times \mathbb{S}^{n-1}(r) \hookrightarrow \mathbb{R}^{n+1}$ has constant principal curvatures given by

$$
\lambda_{1}=0, \quad \lambda_{2}=\cdots=\lambda_{n}=\frac{1}{r} .
$$

In this case,

$$
H=\frac{n-1}{n r} \quad \text { and } \quad|\Phi|^{2}=\frac{n-1}{n r^{2}} .
$$

Finally, when $c=-1$, for a given radius $r>0, \mathbb{M}^{1}\left(-\sqrt{1+r^{2}}\right) \times \mathbb{S}^{n-1}(r) \hookrightarrow \mathbb{H}^{n+1}$ has constant principal curvatures given by

$$
\lambda_{1}=\frac{r}{\sqrt{1+r^{2}}}, \quad \lambda_{2}=\cdots=\lambda_{n}=\frac{\sqrt{1+r^{2}}}{r} .
$$

Thus, in this case,

$$
H=\frac{n r^{2}+(n-1)}{n r \sqrt{1+r^{2}}} \quad \text { and } \quad|\Phi|^{2}=\frac{n-1}{n r^{2}\left(1+r^{2}\right)} .
$$

To finish our proof, we use (2-11) and verify with algebraic computations that in all these situations we must have $r=\sqrt{(n-2) /(n R)}$. 
Using the inequality (3-13) and taking into account that the operator $L$ is selfadjoint relative to the $L^{2}$ inner product of the hypersurface $M^{n}$, we also get the following result:

Corollary 3.4. Let $M^{n}$ be a compact linear Weingarten hypersurface immersed in a real space form $\mathbb{Q}_{c}^{n+1}, n \geq 3$, such $R=a H+b$ with $(n-1) a^{2}+4 n(b-c) \geq 0$. Suppose that $R>0$ when $c=0$ or $c=-1$, and that $R>(n-2) / n$ when $c=1$. If

$$
\sup _{M}|\Phi|^{2}<\frac{n(n-1) R^{2}}{(n-2)(n R-(n-2) c)},
$$

then $|\Phi| \equiv 0$ and $M^{n}$ is isometric to $\mathbb{S}^{n}$, up to scaling.

\section{Acknowledgements}

The second author is partially supported by CAPES/CNPq, Brazil, grant Casadinho/Procad 552.464/2011-2. The authors would like to thank the referee for giving some valuable suggestions which improved the paper.

\section{References}

[Alencar and do Carmo 1994] H. Alencar and M. do Carmo, "Hypersurfaces with constant mean curvature in spheres", Proc. Amer. Math. Soc. 120:4 (1994), 1223-1229. MR 94f:53108 Zbl 0802.53017 [Alías and García-Martínez 2010] L. J. Alías and S. C. García-Martínez, "On the scalar curvature of constant mean curvature hypersurfaces in space forms", J. Math. Anal. Appl. 363:2 (2010), 579-587. MR 2011c:53122 Zbl 1182.53052

[Alías et al. 2012] L. J. Alías, S. C. García-Martínez, and M. Rigoli, “A maximum principle for hypersurfaces with constant scalar curvature and applications", Ann. Global Anal. Geom. 41:3 (2012), 307-320. MR 2886200 Zbl 1237.53044

[Brasil et al. 2010] A. Brasil, Jr., A. G. Colares, and O. Palmas, "Complete hypersurfaces with constant scalar curvature in spheres", Monatsh. Math. 161:4 (2010), 369-380. MR 2012e:53112 Zbl 1201.53068

[Cartan 1938] É. Cartan, "Familles de surfaces isoparamétriques dans les espaces à courbure constante”, Ann. Mat. Pura Appl. 17:1 (1938), 177-191. MR 1553310 Zbl 0020.06505

[Cheng and Yau 1977] S. Y. Cheng and S. T. Yau, "Hypersurfaces with constant scalar curvature", Math. Ann. 225:3 (1977), 195-204. MR 55 \#4045 Zbl 0349.53041

[Levi-Civita 1937] T. Levi-Civita, "Famiglie di superficie isoparametriche nell'ordinario spazio euclideo”, Atti Accad. Naz. Lincei, Rend., VI. Ser. 26 (1937), 355-362. Zbl 0018.08702 JFM 63.1223.01

[Li 1996] H. Li, "Hypersurfaces with constant scalar curvature in space forms", Math. Ann. 305:4 (1996), 665-672. MR 97i:53073 Zbl 0864.53040

[Li 1997] H. Li, "Global rigidity theorems of hypersurfaces”, Ark. Mat. 35:2 (1997), 327-351. MR 98j:53074 Zbl 0920.53028

[Li et al. 2009] H. Li, Y. J. Suh, and G. Wei, "Linear Weingarten hypersurfaces in a unit sphere", Bull. Korean Math. Soc. 46:2 (2009), 321-329. MR 2010b:53111 Zbl 1165.53361

[Okumura 1974] M. Okumura, "Hypersurfaces and a pinching problem on the second fundamental tensor”, Amer. J. Math. 96 (1974), 207-213. MR 50 \#5701 Zbl 0302.53028 
[Omori 1967] H. Omori, "Isometric immersions of Riemannian manifolds", J. Math. Soc. Japan 19 (1967), 205-214. MR 35 \#6101 Zbl 0154.21501

[Pigola et al. 2005] S. Pigola, M. Rigoli, and A. G. Setti, Maximum principles on Riemannian manifolds and applications, Mem. Amer. Math. Soc. 822, American Mathematical Society, Providence, 2005. MR 2006b:53048

[Segre 1938] B. Segre, "Famiglie di ipersuperficie isoparametriche negli spazi euclidei ad un qualunque numero di dimensioni”, Atti Accad. Naz. Lincei, Rend., VI. Ser. 27 (1938), 203-207. Zbl 0019.18403

[Shu 2007] S. Shu, "Complete hypersurfaces with constant scalar curvature in a hyperbolic space", Balkan J. Geom. Appl. 12:2 (2007), 107-115. MR 2008e:53096 Zbl 1135.53039

[Shu 2010] S. Shu, "Linear Weingarten hypersurfaces in a real space form", Glasg. Math. J. 52:3 (2010), 635-648. MR 2011f:53136 Zbl 1203.53059

[Yau 1975] S. T. Yau, "Harmonic functions on complete Riemannian manifolds", Comm. Pure Appl. Math. 28 (1975), 201-228. MR 55 \#4042 Zbl 0291.31002

Received June 29, 2012. Revised August 24, 2012.

CícERo P. AQUiNo

DePartamento de Matemática

Universidade Federal do Piauí

64049-550 TERESINA, PiAuí

BRAZIL

cicero@ufpi.edu.br

HENRiQue F. DE Lima

Departamento de Matemática e Estatística

UniVERSIDAdE FEDERAL DE CAMPINA GRANDE

58429-970 CAMPINA GRANDE, PARAÍBA

BRAZIL

henrique@dme.ufcg.edu.br

MARco A. L. VelásQuez

Departamento de Matemática e Estatística

UNIVERSIDADE FEDERAL DE CAMPINA GRANDE

58.429-970 CAMPINA GRANDE, PARAÍBA

BRAZIL

marco.velasquez@pq.cnpq.br 


\title{
PACIFIC JOURNAL OF MATHEMATICS
}

\author{
msp.org/pjm
}

Founded in 1951 by E. F. Beckenbach (1906-1982) and F. Wolf (1904-1989)

\section{EDITORS}

V. S. Varadarajan (Managing Editor)

Department of Mathematics

University of California

Los Angeles, CA 90095-1555

pacific@math.ucla.edu

Paul Balmer

Department of Mathematics

University of California

Los Angeles, CA 90095-1555

balmer@math.ucla.edu

Daryl Cooper

Department of Mathematics

University of California

Santa Barbara, CA 93106-3080 cooper@math.ucsb.edu

Jiang-Hua $\mathrm{Lu}$

Department of Mathematics

The University of Hong Kong

Pokfulam Rd., Hong Kong jhlu@maths.hku.hk
Don Blasius

Department of Mathematics University of California

Los Angeles, CA 90095-1555

blasius@math.ucla.edu

Robert Finn

Department of Mathematics Stanford University

Stanford, CA 94305-2125

finn@math.stanford.edu

Sorin Popa

Department of Mathematics

University of California

Los Angeles, CA 90095-1555

popa@math.ucla.edu

Paul Yang

Department of Mathematics

Princeton University

Princeton NJ 08544-1000

yang@math.princeton.edu

\section{PRODUCTION}

Silvio Levy, Scientific Editor, production@msp.org

\section{SUPPORTING INSTITUTIONS}

ACADEMIA SINICA, TAIPEI

CALIFORNIA INST. OF TECHNOLOGY

INST. DE MATEMÁTICA PURA E APLICADA

KEIO UNIVERSITY

MATH. SCIENCES RESEARCH INSTITUTE

NEW MEXICO STATE UNIV.

OREGON STATE UNIV.

\author{
STANFORD UNIVERSITY \\ UNIV. OF BRITISH COLUMBIA \\ UNIV. OF CALIFORNIA, BERKELEY \\ UNIV. OF CALIFORNIA, DAVIS \\ UNIV. OF CALIFORNIA, LOS ANGELES \\ UNIV. OF CALIFORNIA, RIVERSIDE \\ UNIV. OF CALIFORNIA, SAN DIEGO \\ UNIV. OF CALIF., SANTA BARBARA
}

\author{
Vyjayanthi Chari \\ Department of Mathematics \\ University of California \\ Riverside, CA 92521-0135 \\ chari@math.ucr.edu \\ Kefeng Liu \\ Department of Mathematics \\ University of California \\ Los Angeles, CA 90095-1555 \\ liu@math.ucla.edu \\ Jie Qing \\ Department of Mathematics \\ University of California \\ Santa Cruz, CA 95064 \\ qing@cats.ucsc.edu
}

These supporting institutions contribute to the cost of publication of this Journal, but they are not owners or publishers and have no responsibility for its contents or policies.

See inside back cover or msp.org/pjm for submission instructions.

The subscription price for 2013 is US \$400/year for the electronic version, and \$485/year for print and electronic.

Subscriptions, requests for back issues and changes of subscribers address should be sent to Pacific Journal of Mathematics, P.O. Box 4163, Berkeley, CA 94704-0163, U.S.A. The Pacific Journal of Mathematics is indexed by Mathematical Reviews, Zentralblatt MATH, PASCAL CNRS Index, Referativnyi Zhurnal, Current Mathematical Publications and the Science Citation Index.

The Pacific Journal of Mathematics (ISSN 0030-8730) at the University of California, c/o Department of Mathematics, 798 Evans Hall \#3840, Berkeley, CA 94720-3840, is published monthly except July and August. Periodical rate postage paid at Berkeley, CA 94704, and additional mailing offices. POSTMASTER: send address changes to Pacific Journal of Mathematics, P.O. Box 4163, Berkeley, CA 94704-0163.

PJM peer review and production are managed by EditFLOW ${ }^{\circledR}$ from Mathematical Sciences Publishers.

PUBLISHED BY

mathematical sciences publishers

nonprofit scientific publishing

http://msp.org/

(C) 2013 Mathematical Sciences Publishers 


\section{PACIFIC JOURNAL OF MATHEMATICS}

Volume $261 \quad$ No. $1 \quad$ January 2013

Hierarchies and compatibility on Courant algebroids

Paulo Antunes, Camille Laurent-GengouX and

JoANA M. NunES DA COSTA

A new characterization of complete linear Weingarten hypersurfaces in real 33 space forms

Cícero P. Aquino, Henrique F. DE Lima and

MARCO A. L. VELÁSQUEZ

Calogero-Moser versus Kazhdan-Lusztig cells

CÉDRIC BONNAFÉ and RAPHAËL ROUQUIER

Coarse median spaces and groups

BRIAN H. BOWDITCH

Geometrization of continuous characters of $\mathbb{Z}_{p}^{\times}$

CLIFTON CUNNINGHAM and MASOUd KAMGARPOUR

A note on Lagrangian cobordisms between Legendrian submanifolds of $\mathbb{R}^{2 n+1}$

\section{ROMAN GOLOVKO}

On slope genera of knotted tori in 4-space

Yi LIU, Yi Ni, HoNGBIN SUN and SHICHENG WANG

Formal groups of elliptic curves with potential good supersingular reduction

$$
\text { ÁlVARO LOZANO-ROBLEDO }
$$

Codimension-one foliations calibrated by nondegenerate closed 2-forms

DAVID MARTínez TORRES

The trace of Frobenius of elliptic curves and the $p$-adic gamma function

DERMOT MCCARTHY

$(D N)-(\Omega)$-type conditions for Fréchet operator spaces 